\title{
In-Flight Evaluation of the Traffic Aware Planner on the NASA HU-25A Guardian Aircraft
}

\author{
Matthew C. Underwood, ${ }^{1}$ Timothy A. Lewis, ${ }^{1}$ and Terique L. Barney ${ }^{2}$ \\ NASA Langley Research Center, Hampton, VA 23681, USA
}

\begin{abstract}
NASA's Traffic Aware Planner (TAP) software is a research-prototype decision support tool that provides pilots with time- and fuel-saving route recommendations that optimize their current trajectory. The software runs on a first-of-a-kind system architecture onboard three aircraft in revenue service conducting operational evaluations with a major domestic airline. Therefore, significant NASA-internal testing is required prior to releasing the software to the partner airline. This paper describes a flight test plan that exercises the functionality of the TAP software in a representative operational environment, describes the system architecture developed and implemented for the NASA Langley HU-25A Guardian aircraft to support the test objectives, presents outcomes of the flight test campaign, and discusses use cases that demonstrate the value of flight testing for this activity.
\end{abstract}

\section{Introduction}

Research into flight path optimization of transport aircraft conducted by the National Aeronautics and Space Administration (NASA) has produced an operational concept known as Traffic Aware Strategic Aircrew Requests (TASAR) [1, 2]. This near-term concept [3] provides the aircrew with a flight deck decision support tool known as the Traffic Aware Planner (TAP). The TAP software leverages a growing number of information sources on the flight deck to make time- and fuel-saving route optimization recommendations to the aircrew while en route. The aircrew can then use the suggestions provided by the tool to make route change requests with a greater likelihood of acceptance by air traffic control (ATC). Since TASAR is a concept intended for the current operational environment, it is intentionally designed to have no safety-critical impact or require any changes to current Federal Aviation Administration (FAA) rules and procedures [4, 5].

The research prototype TAP system [6-8], explained further in Section III.C, continually incorporates up-to-date aircraft state data from onboard avionics, as well as the latest position of surrounding traffic, the most recent wind forecast, and the most recent convective weather forecast, in order to calculate candidate trajectory modifications that improve upon the current active route. These trajectories account for user-selectable objective functions [3] of reduced fuel burn, reduced flight time, or an airline-derived combination of factors known as trip cost. Previous analyses and simulations have estimated substantial savings for airlines employing this technique within the U.S. National Airspace System (NAS) [9-11]. Operational evaluations with Alaska Airlines seek to validate these projected benefits using measured data while simultaneously providing benefits to the airline [12, 13].

The TAP software has undergone a number of human-in-the-loop simulations [14] and flight test activities [15-17] in order to validate the operational concept, evaluate human factors considerations (e.g., workload, usability, distraction, etc.), and to assess the ability of the software to function in a representative operational environment (e.g., connected to live avionics data, using in-flight internet connectivity, etc.). However, these simulations and flight test campaigns did not account for the hardware architecture implemented on the three aircraft for Alaska Airlines' operational evaluations of the TAP software. Therefore, a need was identified to thoroughly test the functionality of the software in a similar hardware architecture to that of the partner airline's aircraft. Information regarding test apparatus and environments used to evaluate TAP prior to testing on the HU-25A can be found in reference [18].

A campaign of flight trials on a NASA aircraft, the HU-25A Guardian, was conducted to ensure that the research prototype TAP system functions well in a configuration similar to the Alaska Airlines aircraft prior to deployment. This airborne, networked environment enables an assessment of the operational factors unique to the flight

\footnotetext{
${ }_{1}^{1}$ Aerospace Engineer, Crew Systems and Aviation Operations Branch, M/S 152, AIAA Member.

${ }^{2}$ Aerospace Engineer, Crew Systems and Aviation Operations Branch, M/S 152.
} 
environment. Additionally, this activity evaluated the effectiveness and benefit of new TAP functionality and operation in a relevant flight environment while allowing the rapid prototyping of new concepts and features.

This paper is organized as follows: Section II discusses the details of the flight test plan, flight profiles, and the duties of personnel involved with conducting flight operations. Section III describes the test platform, avionics equipage, and system architecture. Section IV presents a discussion of results, and Section V contains concluding remarks.

\section{Flight Test Plan}

An 80-hour flight evaluation program was planned that advanced in incremental steps of increasing complexity. The first flights established basic data connectivity to avionics systems and external data sources via in-flight internet. Once the software system was integrated with the aircraft, flights proceeded through system functionality verification testing, and culminated in the operational use of TAP to generate and display trajectory optimizations to the user.

\section{A. Profile Types}

The flight test campaign was conducted in the eastern half of the United States. Evaluation routes were developed based on published Instrument Flight Rules (IFR) navigation aids and fixes. Cruise altitudes varied from Flight Level (FL) 200 through FL380, based on weather conditions. Typical flights followed one of two profiles: "roundrobin" or "out-and-back". Flights were planned to maximize the probability of getting clearance from ATC to fly the filed flight plan. The team avoided flying in the northeast region since, based on prior experiences, the research team was cognizant of the fact that any non-normal routing was likely to be modified by ATC.

\section{Round-Robin Profile}

The round-robin profiles constituted the majority of the test program. These flights originated and terminated at Langley Air Force Base (KLFI), adjacent to NASA Langley Research Center, and proceeded through a number of en route waypoints. The round-robin profile was designed to minimize the duration of the flight day while presenting an opportunity to conduct most types of testing. ${ }^{1}$ An example of a full round-robin route is presented in Fig. 1.

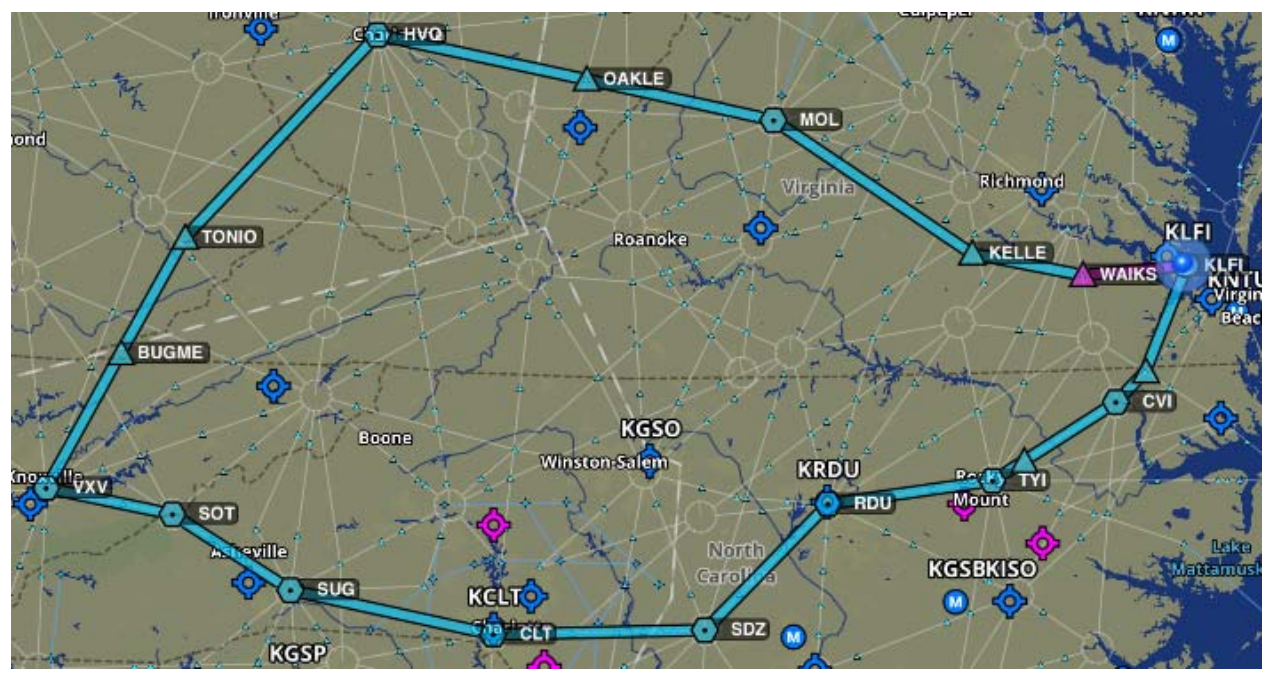

Fig. 1 Round-Robin Profile Example

\section{Out-and-Back Profile}

The out-and-back profile closely replicated routes typically flown by commercial airline operators; however, these profiles could only be flown on a few occasions during the test program due to the long hours required to accomplish them. Figure 2 displays an example of an outbound leg (a) and an inbound leg (b). This particular example of the

\footnotetext{
${ }^{1}$ The TAP software is not designed to work with "round-robin" flight plans due to the origin and destination airports being identical, which is atypical of the vast majority of airline operations. Therefore, to operate the TAP software on this type of flight path, a separate outbound leg and inbound leg were created in the ship's flight management system (FMS) for each round-robin flight plan, and each leg terminated at an airfield.
} 
profile lands at Jacksonville International Airport (KJAX) on the outbound leg, then returns to KLFI on the inbound leg.

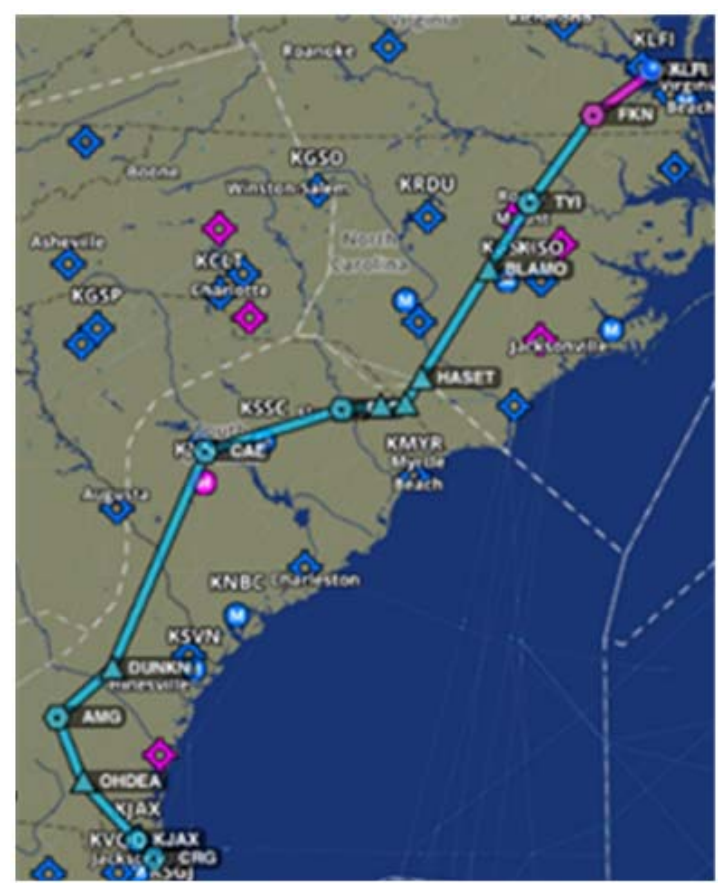

(a) Outbound Leg Example

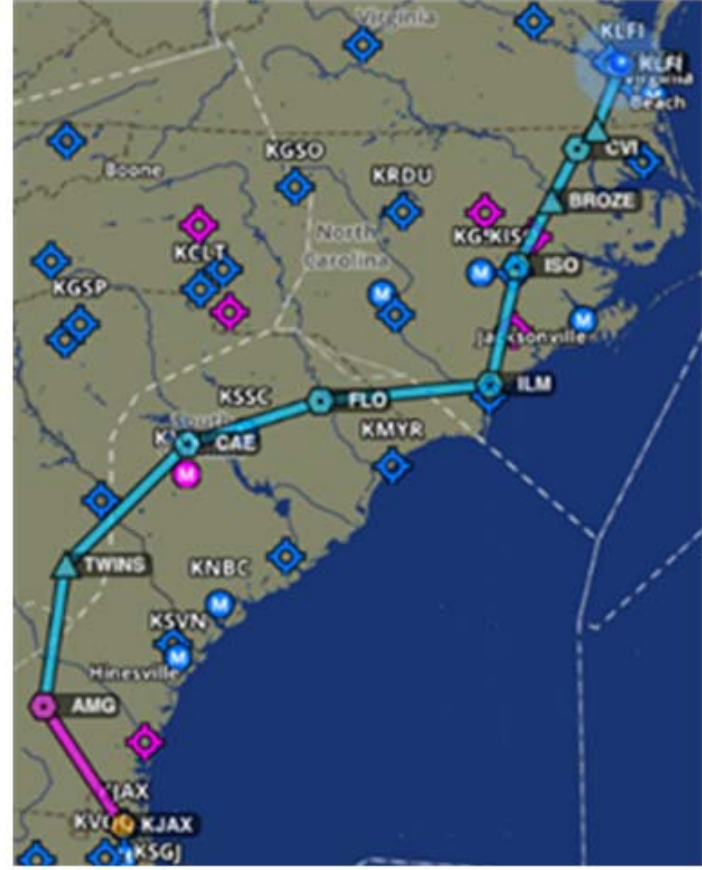

(b) Inbound Leg Example

Fig. 2 Out-and-Back Profile Example

\section{B. Flight Planning Approach}

Flight planning considered specific test objectives for that flight, e.g., altitudes that would provide desired wind conditions, flight time, and range of the aircraft. Additionally, many flights were intentionally planned near convective weather to test the ability of the TAP software to provide a conflict-free trajectory optimization in the presence of weather. Commercially available flight planning software was used to create the flight plans.

To properly test the ability of the TAP software to optimize an aircraft's trajectory, the research team intentionally introduced inefficiencies in the flight plans for each test. Figure 2 demonstrates an example of an introduced inefficiency. Instead of the outbound leg continuing down the east coast, a westerly turn was introduced north of Myrtle Beach, SC, that brought the route inland before proceeding to KJAX. This flight plan inefficiency provided TAP with an opportunity for optimization and the opportunity for the research team to test the associated functionality. Testing the benefit outcomes of the software, the pilot's acceptability of the software, and ATC acceptance of the aircrew's queries in a realistic and relevant operational environment will occur on the Alaska Airlines aircraft during the operational evaluation [13].

Creating a deliberately inefficient yet still operationally representative flight plan that mimicked profiles flown by commercial airliners was a non-trivial task. Furthermore, planning a route that exercised a key capability of the TAP optimization algorithm, namely the ability to find a non-intuitive, wind-optimal route, was difficult to do manually. Therefore, an approach was devised to plan realistic inefficient routes using the NASA Langley Air Traffic Operations Simulation (ATOS) software [19] in conjunction with TAP. Several scenarios were created in ATOS that featured great circle (i.e., shortest flight path) routes between KLFI and airports to the north, northwest, west, southwest, and south using a performance model of the HU-25A Guardian. Table 1 identifies airports that were considered. Various directions from KLFI were chosen to expose TAP to multiple opportunities to find wind-optimal routes.

Table 1 Flight Planning Airports

\begin{tabular}{|c|c|c|c|}
\hline Direction from KLFI & Airport Name & Airport Code & City/State \\
\hline \hline North & Burlington International Airport & KBTV & Burlington, VT \\
\hline Northwest & Gerald R. Ford International Airport & KGRR & Grand Rapids, MI \\
\hline West & Wichita Dwight D. Eisenhower National Airport & KICT & Wichita, KS \\
\hline
\end{tabular}




\begin{tabular}{|c|c|c|c|}
\hline Southwest & Louis Armstrong New Orleans International Airport & KMSY & New Orleans, LA \\
\hline South & Jacksonville International Airport & KJAX & Jacksonville, FL \\
\hline
\end{tabular}

On the morning of a flight, these scenarios were ran in ATOS and the TAP software was connected to a simulated aircraft. The TAP software downloaded the latest wind and weather data, and provided optimized trajectory solutions based on the route in each scenario and the current wind and weather data. The solutions were used to define what TAP thought the most optimal route was, and a representative flight plan was created using the great circle route.

For example, the Flight Test Director generated and started an ATOS scenario using the HU-25A performance model based on a great circle route between KLFI and KICT. The TAP software connected to the simulated aircraft as shown in Fig. 3, and provided a non-intuitive wind-optimal route (notice the strong headwinds along the magenta route, and the lesser headwind to the right of the magenta route). This process verified that the great circle route was realistic yet inefficient due to the winds at altitude. In this case, the great circle route became the filed flight plan, demonstrating this effectiveness of this flight planning technique.

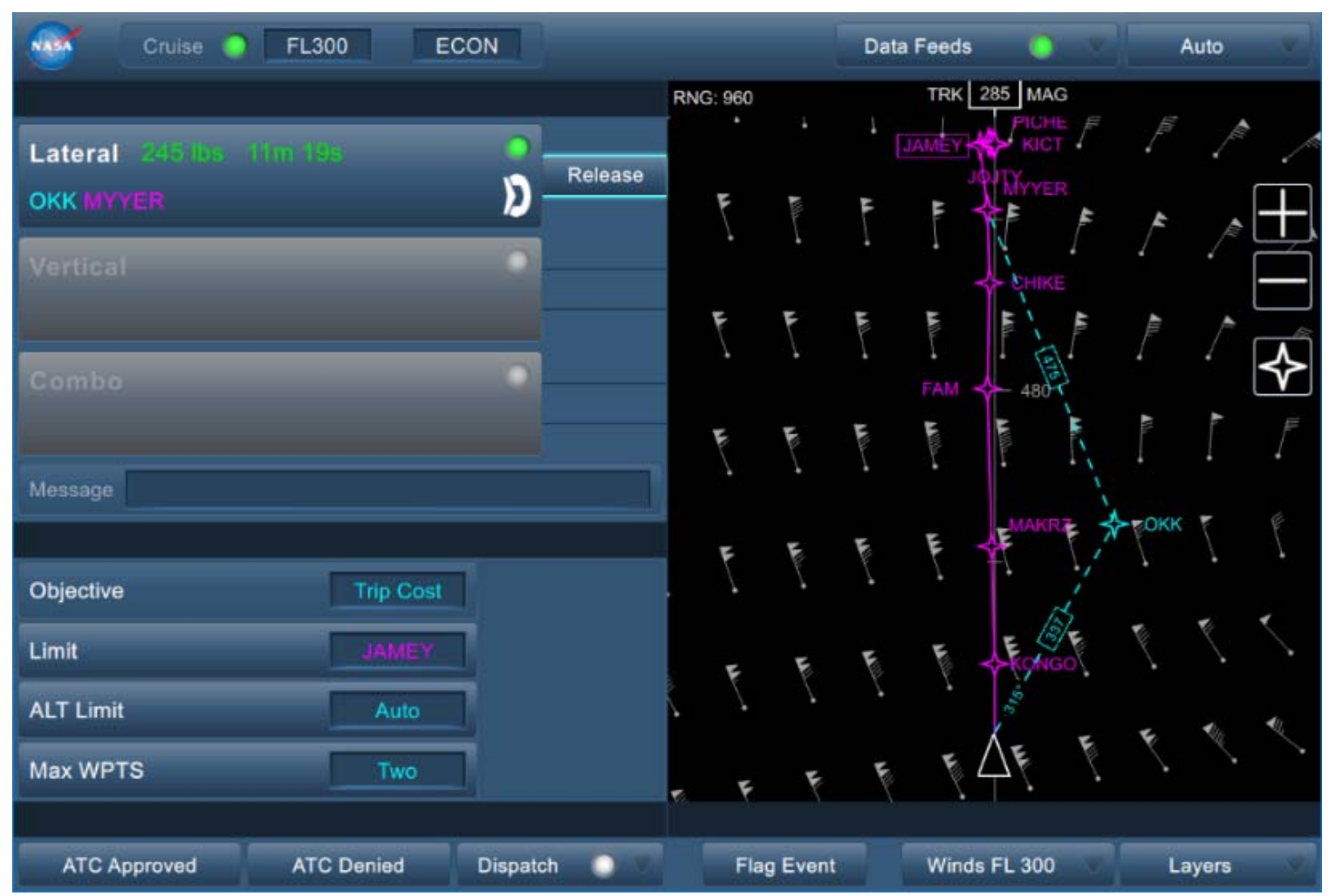

Fig. 3 Using the TAP Software for Flight Planning

\section{Duties of Personnel}

Several individuals were required to ensure that each flight was successful. The roles and responsibilities of those directly involved with the research effort (aircrew, cabin crew, and ground research support) are described below.

\section{Aircrew}

The aircrew consisted of two NASA pilots, who did not interact with the TAP software directly; their role was to assure the safety of the flight and to ensure that objectives were met from a flight conduct perspective. The aircrew also programmed the aircraft's avionics that fed the route to the TAP software, and ensured that the vehicle was flying in an appropriate guidance mode for the TAP software to provide optimized trajectory solutions.

\section{Flight-based research team}

The flight-based research team nominally consisted of four to six personnel: a Flight Test Director, a Flight Test Communications Manager, and two to four TAP Software Testers.

Prior to the flight, the Flight Test Director generated the flight plan, coordinated the flight manifest with the research team and aircrew, and conducted a pre-flight briefing outlining the details of the flight. During the flight, the 
Flight Test Director was the conduit for all TAP-related internal aircraft communications with the NASA aircrew, as well as responsible for ensuring that tests points were conducted in the proper order. After each flight test, the Flight Test Director conducted a post-flight debrief and communicated the debrief outcomes to the research and development teams.

The Flight Test Communications Manager handled communications between the flight-based research team and the ground-based research team. This communication was conducted via Satellite Communication (SATCOM) phone calls, SATCOM text messaging, or internet-based text messaging. TAP Software Testers were responsible for conducting TAP functional requirements verification using systematic test procedures outlined in the TAP software test cards or performing unstructured functionality testing. On several flights, other personnel (e.g., subject-matter experts, representatives from Alaska Airlines, project personnel, etc.) observed TAP testing, operated TAP in the aircraft, and provided feedback and suggestions regarding the tool.

\section{Ground-based research and development team}

The ground-based research team operated from the NASA Langley Flight Operations Room, where they observed the flight remotely through commercially available flight tracking websites, and correlated commercial weather radar with the research generated weather data used by TAP. The Flight Operations Room also had the capability to connect via Satcom radio to the flight-based research team and via teleconference to remote researchers and developers, thereby allowing instantaneous communication between all parties for trouble-shooting and clarifying lessons learned.

\section{Test Platform}

This section addresses the airborne testing platform, its avionics suite, and hardware installed on the aircraft to enable flight-testing of the TAP software. The system architecture design and implementation for testing the TAP software is also discussed.

\section{A. Flight Test Aircraft}

The flight test vehicle chosen for this activity was the NASA Langley HU-25A Guardian (NASA524, shown in Fig. 4), a modified version of the Dassault FA-20G Falcon. The Guardian has a broad flight envelope representative of the operational environment of a commercial airliner. It is capable of flying up to FL390, has a typical cruise speed of Mach 0.72 at FL320, and a range of over 2,000 nautical miles [20]. The cabin of this aircraft is large enough to accommodate a contingent of seven researchers, along with laptop computers, tablets, and other carry-on equipment.

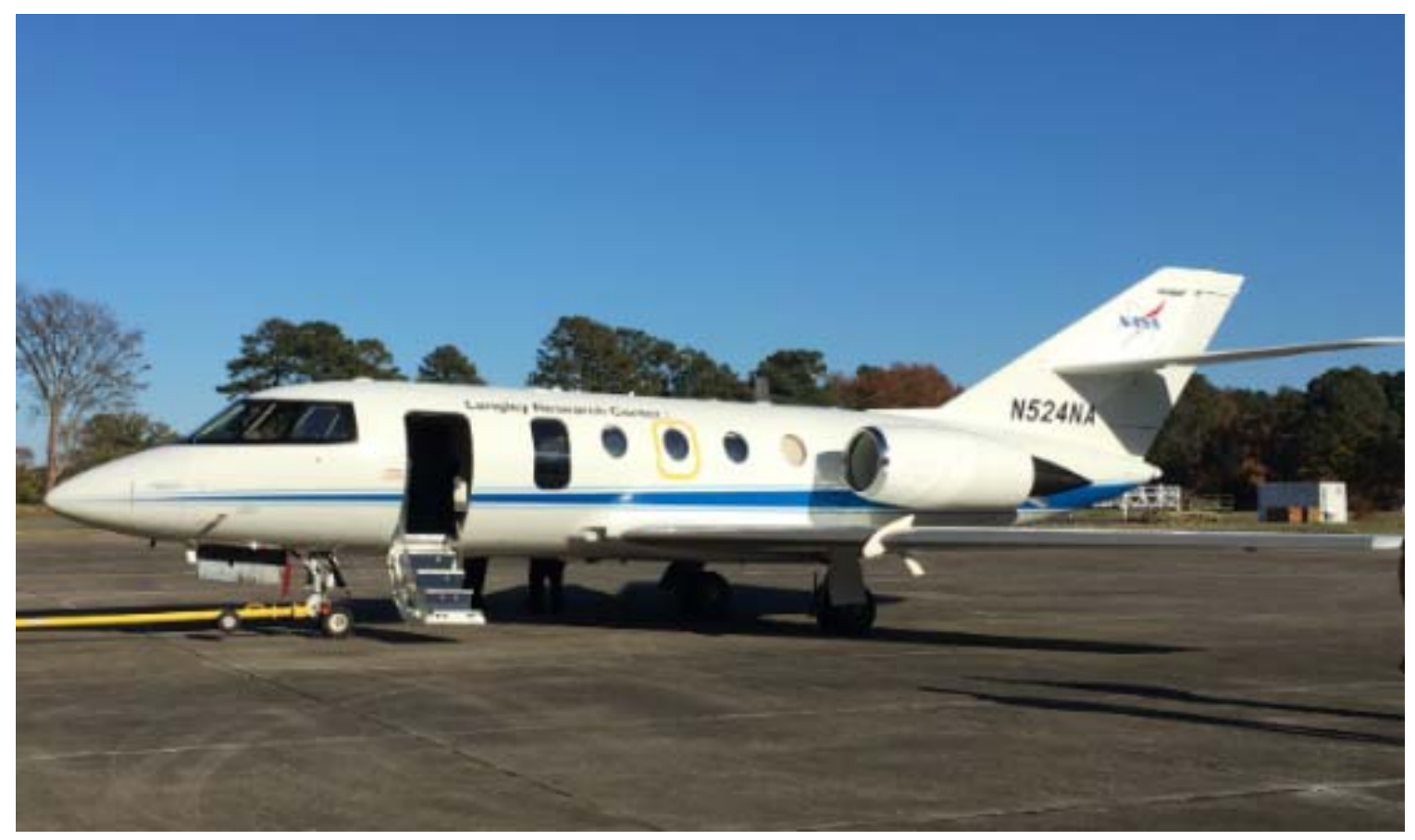

Fig. 4 HU-25A Guardian Aircraft, NASA524 


\section{B. Avionics and Flight Test Hardware ${ }^{1}$}

The HU-25A Guardian is equipped with a baseline set of certified avionics equipment that provided data required for TAP, including:

- Rockwell Collins ADS-80 Air Data System

- Litton LTN-72 Inertial Reference System

- Rockwell Collins CDU-900 Flight Management System

- Garmin GTN-750 Flight Management System and Multifunction Display

- Garmin GDL-88 Dual-band Automatic Dependent Surveillance-Broadcast (ADS-B) System

- Garmin GTX-3000 Datalink Transponder

- Honeywell TPA-100B Enhanced Traffic Collision Avoidance System (TCAS II) Processor

- Teledyne Digital Flight Data Acquisition Unit

Additionally, the HU-25A Guardian is equipped with additional certified equipment specifically for TAP system testing. These included:

- Cobham AVIATOR 200 SATCOM terminal and antenna for in-flight internet and cabin Wi-Fi connectivity

- Rockwell Collins RTA-4118 MultiScan weather radar; connectivity to Ballard ARINC 708 to USB adapter

- Rockwell Collins MFD-255 Multi-Function Display for weather radar display and control

- United Technologies Aerospace Systems (UTAS) Aircraft Interface Device 2 (AID2)

Finally, additional carry-on hardware brought onto the HU-25A Guardian to enable the TAP software testing included:

- One UTAS Tablet Interface Module (TIM)

- Up to four docked Dell Precision 7520 laptop computers

- Apple iPads serving as displays for the TAP software

- Microsoft Surface Pro tablets serving as standalone TAP testing systems

\section{System Architecture}

The goals of the system architecture for the HU-25A test platform were to be inexpensive, adaptable, and portable. Furthermore, as the flight campaign proceeded, an additional design goal emerged to replicate as closely as practical the system architecture of the Alaska Airlines aircraft. This architecture allowed the research team to configure the hardware and network settings in a laboratory environment, run tests to ensure that the configuration functioned properly, and then take the hardware with the lab configuration directly to the aircraft without additional modifications. This resulted in a significant decrease in deployment time to the HU-25A and a decrease in setup time once on the aircraft, creating a more efficient flight testing operation.

\section{Software System Architecture Overview}

The TAP system consists of four executable components that reside on airborne systems: the TAP Engine, the TAP Display, the TAP Display Adapter (TDA), and the External Data Server (EDS). A fifth component, known as the Ground Data Server (GDS), resides on a ground-based system. A high-level description of these components follows.

1) TAP Engine: The TAP Engine is the main processor of TAP. It accepts and reads avionics data inputs, ADS-B In data, data from the EDS (described below), reformats those data as needed for internal use, performs all processing necessary to generate trajectory solutions, and responds to all pilot commands on the TAP Display that affect processing. It consists of a trajectory generator that creates flyable candidate trajectory solutions, a conflict probe that searches for conflicts along those candidate trajectory solutions, and a pattern-based genetic algorithm that resolves any conflicts along the candidate trajectory solutions. It is designed to operate on both Linux and Windows operating systems [7].

2) TAP Display: The TAP Display (shown in Fig. 3) is the human-machine interface that enables interaction between the pilot and the software. It displays up to three optimal trajectory solutions: lateral only, vertical only, and a combination of lateral/vertical maneuvers. It also displays the time and fuel outcomes for each trajectory solution, conflict information, and additional information regarding the internal state of the system. It also

${ }^{1}$ The use of trademarks or names of manufacturers in this report is for accurate reporting and does not constitute an official endorsement, either expressed or implied, of such products or manufacturers by the National Aeronautics and Space Administration. 
accepts all pilot-entered information and sends it to the TAP Engine. It is designed to operate on both Windows and iOS operating systems [7].

3) TAP Display Adapter: The TDA handles all communications between the TAP Engine and the TAP Display. In hardware configurations where the TAP Display is operating on a tablet device, the TDA also reestablishes connections with the TAP Display after the device has been put to sleep or the TAP Display has been pushed into background operation while another application is in use. It is designed to operate on both Linux and Windows operating systems [7].

4) External Data Server: The EDS handles the connection, download, and processing of data obtained via the internet. These data include convective weather data from the GDS, winds data from the National Oceanic and Atmospheric Administration (NOAA), and Special Use Airspace (SUA) data from the FAA. It is designed to operate on both Linux and Windows operating systems [7].

5) Ground Data Server: The GDS handles the downloading and processing of large sets of data obtained via the internet, such as weather data from a third-party vendor. These data include now-cast convective weather avoidance polygons, forecast convective weather avoidance polygons, convective SIGnificant METeorological (SIGMET) data, and turbulence SIGMETs. It is designed to operate on a Linux operating systems [21].

Each TAP airborne component communicates with other components via TCP/IP sockets in a serial manner (i.e., EDS communicates with TAP Engine, Engine communicates with TDA, etc.) as highlighted in Fig. 5. The EDS accesses the external ground data sources (GDS, NOAA Winds, and FAA SUA Activation Schedule) via airborne internet. An ARINC 424 navigation database provides airspace information (waypoints, intersections, SUAs, etc.) that the TAP Engine uses in its computations. The TAP Engine requires state data (position, altitude, speed, weight, etc.) from the aircraft, as well as information about its route and guidance modes. Data from the EDS, as well as the onboard sources is processed in the TAP Engine, and the optimized trajectory solutions are transmitted to the TAP Display via the TDA.

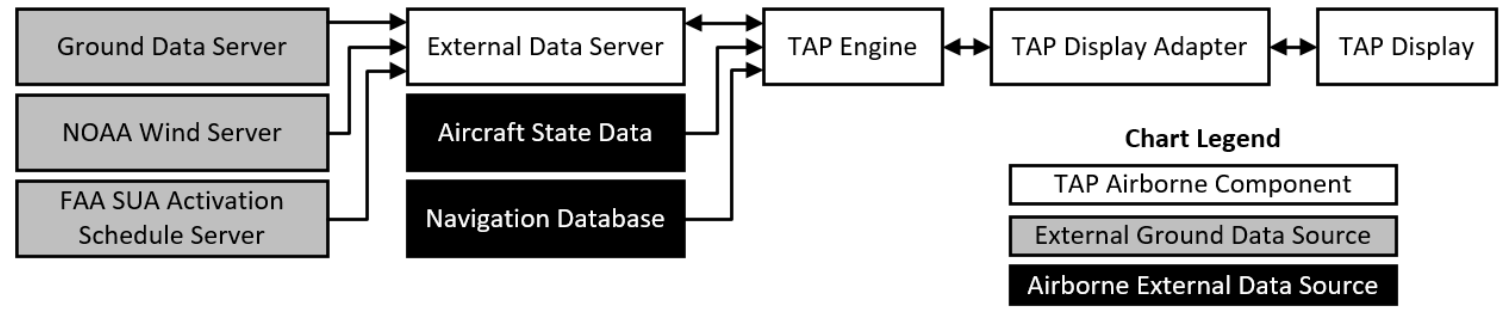

Fig. 5 TAP Software System Architecture

\section{Hardware Configuration and Network Topology}

Figure 6 outlines the hardware configuration and network topology for the HU-25A. The aircraft's certified avionics are connected to a UTAS AID2, which accepts both ARINC 429 and ARINC 717 inputs. The AID2 features an ARINC 834 Simple Text Avionics Protocol (STAP) server, which provides aircraft state and route data in a standard format to entities connected to the server. The AID2 packages the ARINC 429 payloads received from the avionics bus using STAP as defined by the ARINC 834 Aircraft Data Interface Function standard [22] and transmits the data to computing processors on a local network.

The AID2 allows up to six entities to connect to the STAP server via 10/100 Base-T ports. In the system architecture utilized on the HU-25A, the four docked Windows laptops are connected directly to the AID2 via Ethernet. These components are systems that require a robust and reliable hard-wired connection to the STAP server. A UTAS TIM occupies the fifth port- the TIM provides a networking interface between an iPad and the AID2 via Bluetooth. The TIM serves as a network pass-through between the First Officer's (F.O. in Fig. 6) TAP Display application, running on an iPad, and the laptop hosting the First Officer's TDA. The sixth port connects the aircraft's SATCOM terminal to the AID2, which permits other computing platforms in the aircraft's cabin that may be hosting the TAP software to access data from the STAP server via Wi-Fi. Furthermore, the connection between the AID2 and the SATCOM terminal serves as a network bridge that allows computing platforms on the Wi-Fi network to connect to computing platforms on the hard-wired network. For example, an iPad hosting the Captain's (Capt. in Fig. 6) instance of the TAP Display application is able to communicate with a Windows laptop hosting the Captain's instances of the TAP Engine and TAP Display Adapter through this network bridge. 


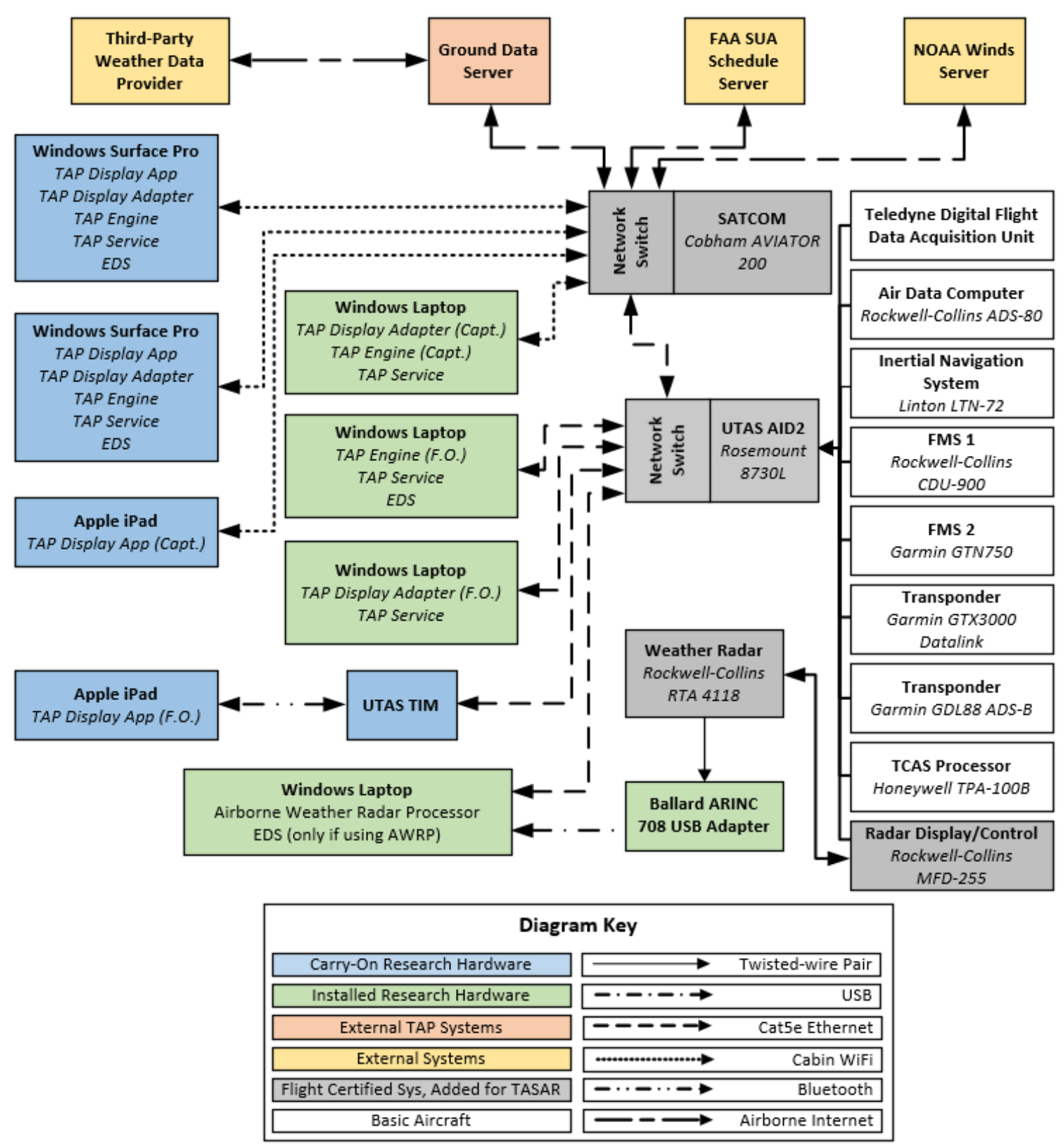

Fig. 6 System Architecture for HU-25A

\section{Nominal HU-25A Test Configuration}

For the majority of flights, four independent TAP systems operated in the cabin of the aircraft. The two primary systems were representative of the Alaska Airlines operating environment - one system replicated the Captain's system operating on a Windows laptop and an Apple iPad and another system replicated the First Officer's system operating on two Windows laptops, a UTAS TIM, and an Apple iPad. The other two systems, predominately used for functional testing and demonstration, were standalone self-contained systems operating on Microsoft Surface Pro tablet computers.

\section{Airborne Weather Radar Research System Configurations}

Early in the flight test campaign, emphasis was placed on obtaining airborne weather radar data and using it in a prototype tool to generate avoidance polygons for the TAP software. On these early flights, ARINC 708 outputs from the airborne weather radar were connected to a Ballard ARINC 708 USB Adapter that allowed for a laptop computer 
to receive and process these data. Discussed further in [21], a prototype tool known as the Airborne Weather Radar Processor (AWRP) ingested these data from the weather radar and used them to build avoidance polygons for the TAP software. This prototype tool provided avoidance polygons to the TAP software on one flight at the end of March 2018.

\section{Flight Evaluation Outcomes}

This section describes a general overview of the flight activity and three case studies of TAP software issues discovered using this flight asset.

\section{A. Overview of Flight Evaluation Results}

The flight testing team conducted 19 flights, flying more than 15,000 nautical miles over approximately 50 flight hours. Figure 7 depicts a sub-set of flight paths flown during the test campaign. Initial flights from August 2017 through March 2018 successfully tested the integration of the TAP system with the HU-25A Guardian, and also collected several hours of data from the prototype AWRP tool. From May through July 2018, flights assessed the performance of the TAP software near convective weather, demonstrated the ability to ingest airborne weather radar data from AWRP in real-time and use it in trajectory optimization (discussed further in [21]). Flight tests during this time period also tested the ability to connect to a ground-based trajectory optimization tool designed for airline dispatchers running at NASA Ames Research Center. From August to December of 2018, flights evaluated the performance of the TAP software on a hardware architecture more closely aligned to that of the Alaska Airlines' aircraft participating in the operational evaluation, and a more formalized and rigorous testing procedure was implemented.

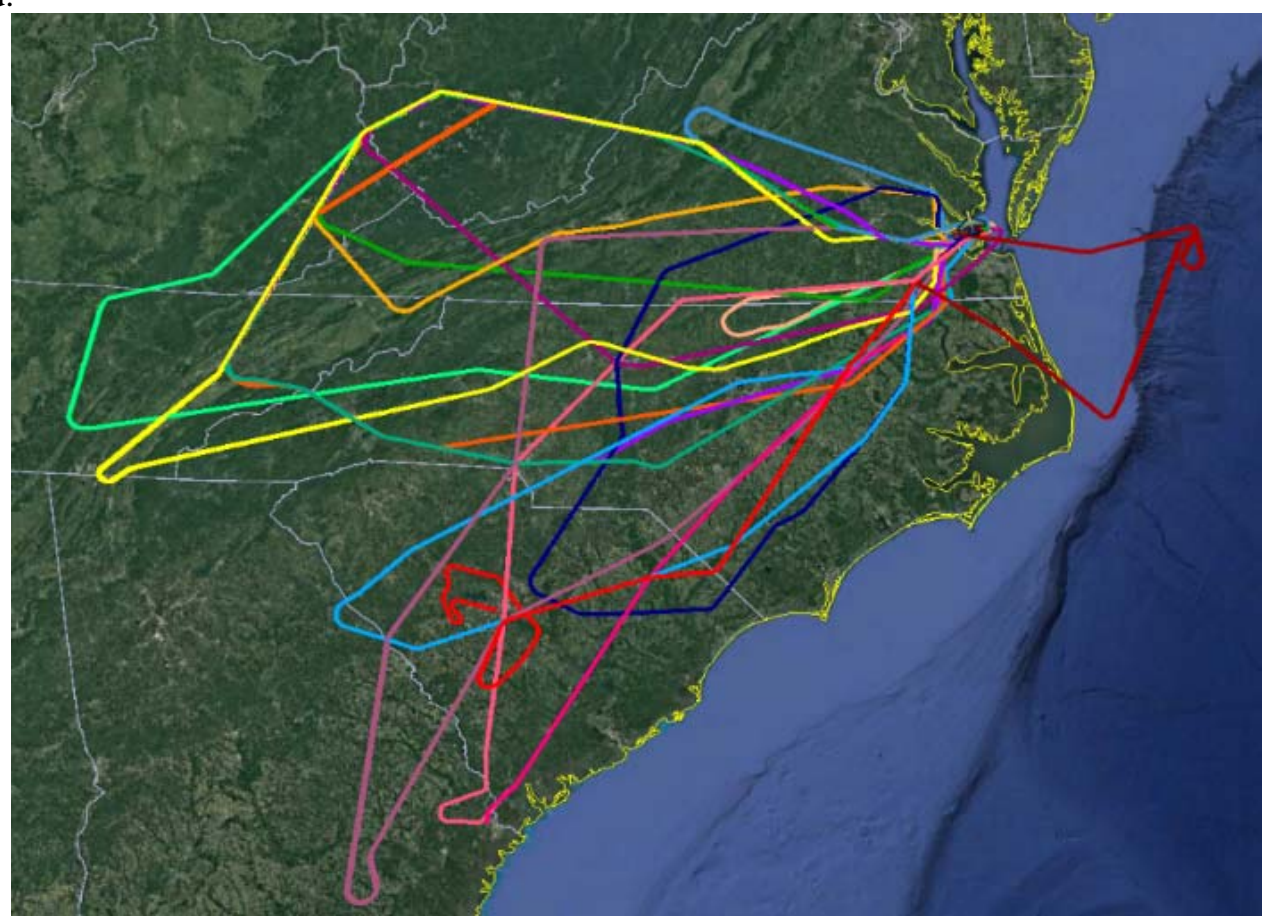

Fig. 7 Example of Flights Conducted as Part of Test Campaign

On several occasions, the flight-based research team requested that the aircrew make a route amendment or altitude change request based on the trajectory solutions provided by the TAP software for proof of concept. Many of these requests were approved by ATC. However, the purpose of the testing was to evaluate the functionality of the software. The operational evaluation with Alaska Airlines, discussed in [13], was designed to test pilot acceptability of the tool, the ATC acceptance rate, and the benefits produced by the tool. On a few occasions, requests were intentionally made when the TAP software indicated that the trajectory solution indicated a traffic conflict. For example, on July 31, 2018, the aircrew, at the request of the flight test director, requested a TAP-suggested descent from FL360 to FL320 to save time. The TAP software provided information to the flight test director that a traffic conflict existed at FL320. The request was denied by ATC because of the traffic conflict. When the TAP software indicated that the traffic was no longer a conflict, the aircrew again requested the descent, and it was approved by ATC. 


\section{B. Case Studies}

This section uses three case studies to illustrate the benefit of having simulation platforms of increasing fidelity, and in particular, the use of high-fidelity flight testing to validate the promising solutions identified during lowerfidelity simulation testing. These case studies highlight an adjustment to the size of the lateral buffer of convective weather polygons, an issue in the trajectory generator regarding the crossover altitude, and a bug in the Display that caused the software to crash.

\section{Polygon Buffer Size Adjustment}

TAP makes use of four-dimensional convective weather avoidance polygons in its trajectory optimization algorithm. These GDS-generated polygons are drawn around areas of hazardous weather conditions, which were defined as areas with a radar reflectivity greater than or equal to $40 \mathrm{dBZ}$ to be consistent with the FAA definition of "Heavy" precipitation intensity [23, 24]. In addition to encompassing the weather phenomena of interest, buffers of twenty nautical mile laterally and 5,000 feet vertically were added initially to the GDS polygons used by TAP. The initial values for the lateral and vertical buffers were set based on trade-offs between conservativism with respect to avoiding the weather, the uncertainty and inaccuracies of weather forecasts, and providing the TAP algorithms adequate space to optimize the route in the vicinity of weather.

The HU-25A encountered convective weather during a test flight on May 16, 2018. The white lines in Fig. 8 show the result of adding a twenty nautical mile buffer to the weather avoidance polygons, with the magenta line showing the flight path flown by the NASA pilots using standard NASA and FAA guidance for weather avoidance. While the weather avoidance polygons correctly matched up to the areas of convective weather greater than or equal to $40 \mathrm{dBZ}$, the lateral bounds of the polygons extended significantly beyond the hazardous weather areas due to the lateral buffer applied to each polygon, causing an inaccurate representation of the weather that the aircraft actually avoided. This exaggerated avoidance area was corroborated through the use of the airborne weather radar in the HU-25A, turbulence felt during the flight, and visual observations by the pilots and researchers. This clearly demonstrated that the lateral buffer parameters set initially using the simulation test environment were too conservative.

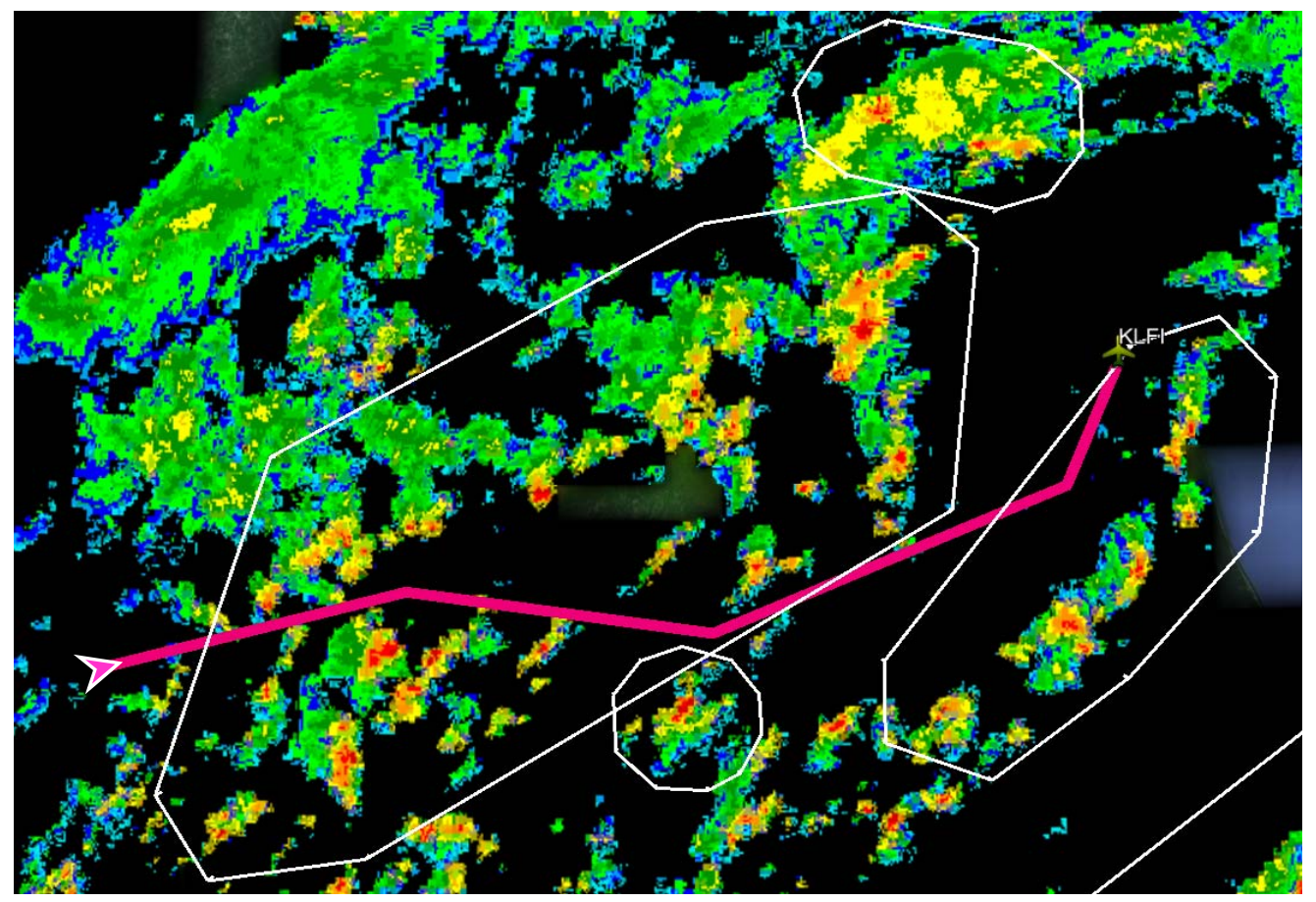

Fig. 8 Portrayal of Inaccurate Avoidance Polygons due to Large Lateral Buffers

A post-flight analysis was conducted to determine the effects of different lateral buffers on the weather avoidance polygons. This analysis, supported by pilot subject matter expertise, focused on refining the value for the lateral avoidance buffer that best ensured avoidance of the weather while maintaining a realistic representation of the convective weather hazard. Three values for the lateral buffer were examined: five nautical miles, ten nautical miles, and twenty nautical miles. 
After consideration from several subject matter expert pilots, including pilots from Alaska Airlines, the lateral buffer was set at five nautical miles. The smaller lateral buffer, according to the subject matter experts, provided the best representation of the weather phenomenon. Furthermore, since the TAP software was not designed as a weather avoidance tool, subject matter experts felt that the best option was to provide the software with the greatest opportunity to optimize the flight in the presence of weather (i.e., use the smallest lateral buffer) and rely on the aircrew to avoid convective weather according to standard operating procedures. The same analysis and discussion led to the final vertical buffer remaining at 5,000 feet. Subsequent flights using the TAP software on both the HU-25A and on Alaska Airlines' aircraft have offered assurance that the five nautical mile lateral buffer, in conjunction with the aircrew maintaining separation from the weather, provides a safe and realistic mechanism for optimizing flights near convective weather.

\section{Mach-Calibrated Airspeed Transition When in Selected Speed Mode}

The crossover altitude is the altitude where a specific Mach number and calibrated airspeed (CAS) value represent the same true airspeed (TAS) value. Above this altitude, Mach number is used to reference speed, and below this altitude, CAS is used to reference speed. At that altitude, a transition from CAS to Mach (ascent) or Mach to CAS (descent) occurs in the trajectory prediction and guidance [25]. The trajectory generator in the TAP software computes a four-dimensional trajectory that takes into account lateral path, vertical profile, and speed along the lateral/vertical flight path. To compute the speed profile, the TAP trajectory generator has two modes-an economy mode (ECON) and a selected speed mode (SEL SPD). The remainder of this section will focus on SEL SPD mode.

In SEL SPD mode at cruise conditions for the ownship trajectory prediction, a constant Mach speed guidance target is used at the cruise altitude. For candidate trajectory solutions with portions of the vertical component existing below the crossover altitude, the trajectory generator nominally uses a constant Mach guidance target during the initial portion of the descent. At the crossover altitude, the speed guidance target switches from constant Mach to a constant CAS. Once the aircraft levels off at the desired cruise altitude, it maintains a constant CAS speed guidance target.

During a flight on May 24, 2018, a pilot SME from Alaska Airlines questioned the accuracy of TAP trajectory solutions with vertical components below the crossover altitude. Analyses revealed that, in these cases, the trajectory generator in TAP never switched the guidance target from constant Mach to constant CAS at the crossover altitude. Because of this behavior, candidate trajectory solutions with a vertical component that descended below the crossover altitude had inaccurate predictions of fuel/time outcomes, thus causing inaccurate optimizations. Assuming that the aircrew will use the TAP software cruise predominantly above the crossover altitude, the most appropriate resolution to ensure that research could continue with the prototype software was to preclude TAP software solutions below FL300.

The majority of commercial airline pilots would utilize TAP in ECON mode, which became the de facto default mode for the majority of simulation testing on the ground. However, the HU-25A is not equipped with an ECON speed flight guidance mode. Therefore, this trajectory generation behavior was uncovered because more thorough testing of the TAP software in SEL SPD mode was conducted onboard the HU-25A. This NASA asset provided an operationally realistic environment within which pilot SMEs could use TAP during actual flight operations, enabling local in-flight demonstration of TAP, valuable end-user feedback, and the identification of software issues such as the one described in this section.

\section{Zero-Groundspeed Display Crash Issue}

During flights on November 14, 2018, the TAP Display crashed several times in a repeatable fashion when the TAP system was launched while the HU-25A was stationary or taxiing slowly. The problem did not manifest when the TAP Display was launched during the takeoff roll or when the aircraft was airborne. Debugging analysis found the root cause was that the TAP Display was not designed to handle a condition of zero airspeed, and in fact, the software logic was such that the error condition was triggered whenever the airspeed is less than or equal to the wind speed, which occurs in a function to compute the groundspeed. The mitigation was to hard-code a minimum ground speed input of 100 knots.

This issue was not uncovered during simulation testing since the ATOS desktop flight simulator used by NASA is not designed for surface operations, and thus the simulated aircraft is always started airborne, which does not excite this bug. The problem was also not observed in any of the operational evaluation data received from the use of TAP on the Alaska Airlines aircraft, although TAP was also launched on the ground while parked at the gate in those tests. This is due to the avionics onboard those aircraft report a minimum airspeed of 40 knots, and so that TAP system never encountered the zero airspeed indication observed by the TAP system on the HU-25A. 


\section{Concluding Remarks}

The TAP software was effectively integrated with the avionics of the HU-25A aircraft, and the software successfully generated realistic candidate trajectory solutions. New, novel methods for planning inefficient flight paths were employed, and several valuable lessons were learned with respect to conducting flight operations. The portable and extensible system configuration implemented on the HU-25A emulated the system architecture of the operational evaluation aircraft flown by Alaska Airlines, while simultaneously maintaining the flexibility to conduct research activities. Because of these flight tests, the NASA TAP software testing team was able to discover and fix software bugs identified uniquely in a flight environment, identify and mitigate issues exposed by using the software in an operationally relevant environment, and provide a level of confidence in the software to end users prior to software deployment to end users.

\section{Acknowledgements}

The authors wish to thank Mike Palmer, Elaine Blount, Mike Day, Doug Mielke, Bob Vivona, Dave Roscoe, Dr. David Karr, Bill O’Neill, Jonathan Starr, Jim Davis, Chris Ware, Rick Maine, Michael Lewis, Tom Britton, Michael Harper, and other members of the Airspace and Traffic Operations Simulation development team for providing software and hardware support; Bill Cotton and Clay Hubbs for their contribution to the operational use of TAP, especially the integration of convective weather avoidance; Matt Elder, Taylor Thorson, Rick Yasky, and Greg Slover for serving as pilots during the flight trials; Kevin Shelton, Ted Wilz, Joseph Riddick, Kevin Hennessey, and other members of the Research Service Directorate for integrating components of the TAP system into and maintaining the test aircraft; Lee Joyce for providing support for the flight operations center; as well as all others who contributed to this effort.

\section{References}

[1] M. G. Ballin and D. J. Wing, "Traffic Aware Strategic Aircrew Requests (TASAR)," in 12th AIAA Aviation Technology, Integration, and Operations Conference; AIAA 2012-5623, Indianapolis, 2012.

[2] J. Henderson, "Traffic Aware Strategic Aircrew Requests (TASAR) Concept of Operations," NASA/CR-2013-218001, Hampton, 2013.

[3] D. J. Wing, M. G. Ballin, S. Koczo, R. A. Vivona and J. M. Henderson, "Developing an Onboard Traffic-Aware Flight Optimization Capability for Near-Term Low-Cost Implementation," in 13th AIAA Aviation Technology, Integration, and Operations Conference; AIAA 2013-4231, Los Angeles, 2013.

[4] S. Koczo, "Analysis of Operational Hazards and Safety Requirements for Traffic Aware Strategic Aircrew Requests," NASA/CR-2013-218002, Hampton, 2013.

[5] S. Koczo, "TASAR Certification and Operational Approval Requirements - Analyses and Results," NASA/CR-2015218708, Hampton, 2015.

[6] S. E. Woods, R. A. Vivona, D. A. Roscoe, B. C. LeFebvre, D. J. Wing and M. G. Ballin, "A Cockpit-based Application for Traffic Aware Trajectory Optimization," in AIAA Guidance, Navigation, and Control Conference; AIAA 2013-4967, Boston, 2013.

[7] S. A. Woods, R. A. Vivona, J. Henderson, D. J. Wing and K. A. Burke, "Traffic Aware Planner for Cockpit-based Trajectory Optimization," in 16th AIAA Aviation Technology, Integration, and Operations Conference; AIAA 2016-4067, Washington, D.C., 2016.

[8] S. A. Woods, "Traffic Aware Strategic Aircrew Requests (TASAR) Analysis and Development Final Report," NASA/CR2016-219197, Hampton, 2016.

[9] J. Henderson, H. Idris and D. A. Wing, "Preliminary Benefits Assessment of Traffic Aware Strategic Aircrew Requests (TASAR)," in 12th AIAA Aviation Technology, Integration, and Operations Conference; AIAA 2012-5684, Indianapolis, 2012.

[10] J. Henderson, "Annualized TASAR Benefit Estimate for Alaska Airlines Operations," NASA/CR-2015-218787, Hampton, 2015.

[11] J. Henderson, "Annualized TASAR Benefit Estimate for Virgin America Operations," NASA/CR-2015-218786, Hampton, 2015.

[12] D. J. Wing, K. A. Burke, J. Henderson, R. A. Vivona and J. Woodward, "Initial Implementation and Operational Use of TASAR in Alaska Airlines Flight Operations," in 18th Aviation Technology, Integration, and Operations Conference, AIAA Aviation Forum; AIAA 2018-3043, Atlanta, 2018.

[13] D. J. Wing, K. A. Burke, K. Ballard, S. R. Wilson, J. Henderson, J. Woodward, "Initial TASAR Operations Onboard Alaska Airlines", in 19th AIAA Aviation Technology, Integration, and Operations Conference; Dallas, 2019. 
[14] D. J. Wing, "Achieving TASAR Operational Readiness," in 15th AIAA Aviation Technology, Integration, and Operations Conference; AIAA 2015-3400, Dallas, 2015.

[15] J. M. Maris, M. A. Haynes, D. J. Wing, K. A. Burke, J. Henderson and S. E. Woods, "Traffic Aware Planner (TAP) Flight Evaluation," in 14th AIAA Aviation Technology, Integration, and Operations Conference; AIAA 2014-2166, Atlanta, 2014.

[16] H. Idris and G. Enea, "TASAR Flight Trial 2: Assessment of Air Traffic Controller Acceptability of TASAR Requests," NASA/CR-2016-219215, Hampton, 2016.

[17] K. A. Burke and M. A. Haynes, "Flight Test Assessments of Pilot Workload, System Usability, and Situation Awareness of TASAR," in Human Factors and Ergonomics Society (HFES) International Annual Meeting, Washington, 2016.

[18] T. L. Barney, M. C. Underwood and B. K. Buck, "Sim-to-Flight Test Environments for the TASAR Traffic Aware Planner," in 19th AIAA Aviation Technology, Integration, and Operations Conference, Dallas, 2019.

[19] D. Finkelsztein, T. Lung, R. A. Vivona, J. Bunnell, D. Mielke and W. Chung, "Airspace and Traffic Operations Simulation for Distributed ATM Research and Development," in AIAA Modeling and Simulation Technologies Conference, San Francisco, CA, 2005.

[20] National Aeronautics and Space Administration, "NASA Airborne Science Program, HU-25A Guardian - LaRC," 25 September 2018. [Online]. Available: https://airbornescience.nasa.gov/aircraft/HU-25A_Guardian_-_LaRC. [Accessed 10 October 2018].

[21] T. A. Lewis, K. A. Burke, M. C. Underwood and D. J. Wing, "Weather Design Considerations for the TASAR Traffic Aware Planner," in 19th AIAA Aviation Technology, Integration, and Operations Conference, Dallas, 2019.

[22] ARINC, "834-3 Aircraft Data Interface Function (ADIF)," ARINC Incorporated, Annapolis, 2012.

[23] Federal Aviation Administration, "ATC Inflight Weather Avoidance Assistance," in Aeronautical Information Manual, Washington, 2014, pp. 7-1-38.

[24] Federal Aviation Administration, "Advisory Circular 00-45H, Aviation Weather Services - Change 1," 14 November 2016. [Online]. Available: https://www.faa.gov/documentLibrary/media/Advisory_Circular/AC_00-45H_Chg_1.pdf. [Accessed 8 March 2019].

[25] Upset Recovery Industry Team, "Airplane Upset Recovery Training Aid Revision 2," Federal Aviation Administration, Washington, D.C., 2008. 